\title{
The value of enhanced CT scanning for predicting lymph node metastasis along the right recurrent laryngeal nerve in esophageal squamous cell carcinoma
}

\author{
Bin $\mathrm{Li}^{1 \#}$, Baiwei $\mathrm{Li}^{2 \#}$, Haoyao Jiang ${ }^{1 \#}$, Yang Yang ${ }^{1}$, Xiaobin Zhang ${ }^{1}$, Yuchen $\mathrm{Su}^{1}$, Rong Hua ${ }^{1}$, Haiyong Gu ${ }^{1}$, \\ Xufeng Guo ${ }^{1}$, Bo Ye ${ }^{1}$, Yu Yang ${ }^{1}$, Yi He ${ }^{1}$, Yifeng Sun ${ }^{1}$, Guillaume Piessen ${ }^{3}$, Steven N. Hochwald ${ }^{4}$, \\ Miguel A. Cuesta ${ }^{5}$, Thomas J. Birdas ${ }^{6}$, Zhigang Li $^{1}$; written on behalf of the AME Thoracic Surgery \\ Collaborative Group
}

${ }^{1}$ Department of Thoracic Surgery, Section of Esophageal Surgery, Shanghai Chest Hospital, Shanghai Jiaotong University, Shanghai, China; ${ }^{2}$ Thoracic Surgery, Tianjin TEDA Hospital, Tianjin, China; ${ }^{3}$ University of Lille, Department of Digestive and Oncological Surgery, Claude Huriez University Hospital, F-59000 Lille, France; ${ }^{4}$ Department of Surgical Oncology, Roswell Park Comprehensive Cancer Center, Buffalo, NY, USA; ${ }^{5}$ Department of Surgery, University Medical Center Amsterdam, Amsterdam, The Netherlands; ${ }^{6}$ Division of Cardiothoracic Surgery, Department of Surgery, Indiana University School of Medicine, Indianapolis, IN, USA

Contributions: (I) Conception and design: Z Li; (II) Administrative support: Z Li; (III) Provision of study materials or patients: Z Li; (IV) Collection and assembly of data: B Li, B Li; (V) Data analysis and interpretation: B Li, B Li, H Jiang; (VI) Manuscript writing: All authors; (VII) Final approval of manuscript: All authors.

\#These authors contributed equally to this work

Correspondence to: Zhigang Li, MD, PhD. Department of Thoracic Surgery, Section of Esophageal Surgery, Shanghai Chest Hospital, Shanghai Jiaotong University, 241 Huaihai West Rd, Shanghai 200030, China. Email: zhigang_li_sch@163.com.

\begin{abstract}
Background: The right recurrent laryngeal nerve (RRLN) is the region most prone to lymph node metastasis in esophageal squamous cell carcinoma (ESCC). Nodal involvement may be underestimated by traditional imaging prediction criteria, such as a short axis diameter of $10 \mathrm{~mm}$. The purpose of this study was to determine a more accurate imaging criterion to guide clinical treatment strategy selection.

Methods: The clinical data of 307 patients with thoracic ESCC who underwent surgery at Shanghai Chest Hospital between January 2018 and December 2018 were retrospectively analyzed. Utilizing 1-mm layer thickness enhanced computed tomography (CT), the RRLN lymph node short diameter (LNSD) size was measured. Univariate and multivariate analyses were performed to determine the risk factors for lymph node metastasis along the RRLN.

Results: In our study, RRLN lymph node metastasis occurred in 60 (19.5\%) patients and general lymph node metastasis occurred in 150 (48.9\%) patients. Of the resected lymph nodes along the RRLN, 14.5\% (121/832) were positive. Multivariate analysis identified LNSD [odds ratio (OR), 1.236] as an independent risk factor for RRLN lymph node metastasis. In CT evaluation, a short diameter of $6.5 \mathrm{~mm}$ in the RRLN lymph nodes is a critical predictor of metastasis at this site (sensitivity $=50 \%$, specificity $=83.4 \%$ ) and a larger short diameter was associated with a higher risk of metastasis $(\mathrm{P}<0.001)$.

Conclusions: A $6.5 \mathrm{~mm}$ cutoff in LNSD can be applied to clinically predict lymph node metastasis in the RRLN region for patients with ESCC.
\end{abstract}

Keywords: Esophageal cancer; lymph node metastasis; recurrent laryngeal nerve; computed tomography (CT)

Submitted Jun 28, 2020. Accepted for publication Dec 09, 2020.

doi: 10.21037/atm-20-4991

View this article at: http://dx.doi.org/10.21037/atm-20-4991 


\section{Introduction}

Esophageal cancer is one of the most common types of malignant tumor, with a 5 -year overall survival rate of a mere $20 \%$ (1). China has the highest incidence and mortality of esophageal cancer anywhere in the world, and squamous cell carcinoma accounts for more than $90 \%$ of those cases (2). Comprehensive treatment with surgery at its core has been considered the mainstream treatment for esophageal cancer, with radical lymph node dissection in particular showing significant survival benefits (3-5). Traditonally, adjuvant chemoradiothearapy following surgery was the mainstream of the treatment. Rencently, the study of CROSS and 5010 have identified the dominance of neoadjuvant chemoradiothearapy for patients with eaophageal cancer. At present, research on immunotherapy is in full swing.

The region along the right recurrent laryngeal nerve (RRLN) is the area most prone to lymph node metastasis in esophageal squamous cell carcinoma (ESCC), with a metastatic probability of between $16.3-20.8 \%(6,7)$. Disease control and staging can be adequately conducted by standardized lymph node dissection (3). However, thorough lymph node dissection along the recurrent laryngeal nerve increases the risk of nerve injury and may lead to the early postoperative complications of aspiration and respiratory insufficiency, further reducing the patient's long-term quality of life $(8,9)$.

Selecting patients who are suitable for lymph node dissection along the RRLN has presented a long-standing clinical problem lacking a clear solution. In last decade, endoscopic dissection has been applied in the treatment of superficial esophageal lesions $(10,11)$. However, endoscopic treatment has no ability to treat involved lymph nodes and preoperative lymph node assessment is especially important when these primary approaches are taken.

Although, in previous clinical studies, the short-diameter of lymph nodes measuring $\geq 1 \mathrm{~cm}$ or axial ratio (shortaxis/long-axis diameters) exceeding 0.66 was used as the diagnostic criterion for metastasis by contrast-enhanced CT scan (12), the actual clinical situation in the area along the RRLN has been significantly underestimated. Therefore, the aim of this study was to establish a clinical standard for predicting lymph node metastasis in the region along the RRLN by retrospectively comparing the detection of RRLN lymph nodes by traditional imaging, as well as pathologic findings, in patients with esophageal squamous cell cancer. This may provide a more accurate staging assessment with relevance to both surgical and non-surgical treatment options.

We present the following article in accordance with the STARD reporting checklist (available at http://dx.doi. org/10.21037/atm-20-4991).

\section{Methods}

\section{Patients}

A retrospective analysis was performed of clinical data from 307 patients with intra-thoracic ESCC who underwent esophagectomy between January 2018 and December 2018 at Shanghai Chest Hospital. The exclusion criteria were: (I) endoscopically diagnosed cervical esophageal cacner or gastroesophageal junction carcinoma, (II) biopsy-confirmed non-squamous cell carcinoma, (III) history of neoadjuvant therapy. The surgical procedure included an Ivor-Lewis or McKeown approach. The study was conducted in accordance with the Declaration of Helsinki (as revised in 2013). This study was approved by the Ethics Committee of Shanghai Chest Hospital (No. KS1734). Because of the retrospective nature of the research, the requirement for informed consent was waived.

All of the patients underwent contrast-enhanced chest and abdomen CT scan, neck ultrasound, esophagogastroduodenoscopy (EGD), and esophageal ultrasonography (EUS) examination. Positron emission tomography/computed tomography (PET/CT) and endoscopic ultrasound with fine-needle aspiration (EUS/ FNA) were selectively used in patients, and bronchoscopy was performed in patients with upper thoracic esophageal cancer.

\section{RRLN lymph node anatomical definition and preoperative image evaluation}

The RRLN node area was defined as follows: the lower boundary was the lower margin of the right subclavian artery, the upper boundary was the cricoid cartilage, the front boundary was the right subclavian artery, and the posterior boundary was the esophagus (Figure 1).

Contrast-enhanced CT scan with 1-mm slice thickness was used to measure the short diameter [lymph node short diameter (LNSD)] of RRLN lymph nodes. The short diameter was calculated using the largest node (Figure 2). The influencing factors were evaluated to establish the predictive factors. 


\section{Right recurrent laryngeal lymph node dissection}

The thoracic phase of the surgery was performed using thoracotomy or video-assisted (alternatively, robot-assisted) thoracoscopic surgery. When right recurrent nerve lymph nodes (106recR) are dissected, protecting the RRLN from damage is essential. Usually, the right inferior thyroid artery is exposed, and the dissection continues until the RRLN is observed as being parallel to the cervical esophagus and entering into the cricothyroid joint.

According to the Esophagectomy Complications Consensus Group (ECCG), recurrent laryngeal nerve injury was defined as unilateral or bilateral vocal cord dysfunction post-resection, as confirmed by endoscopic examination (13).

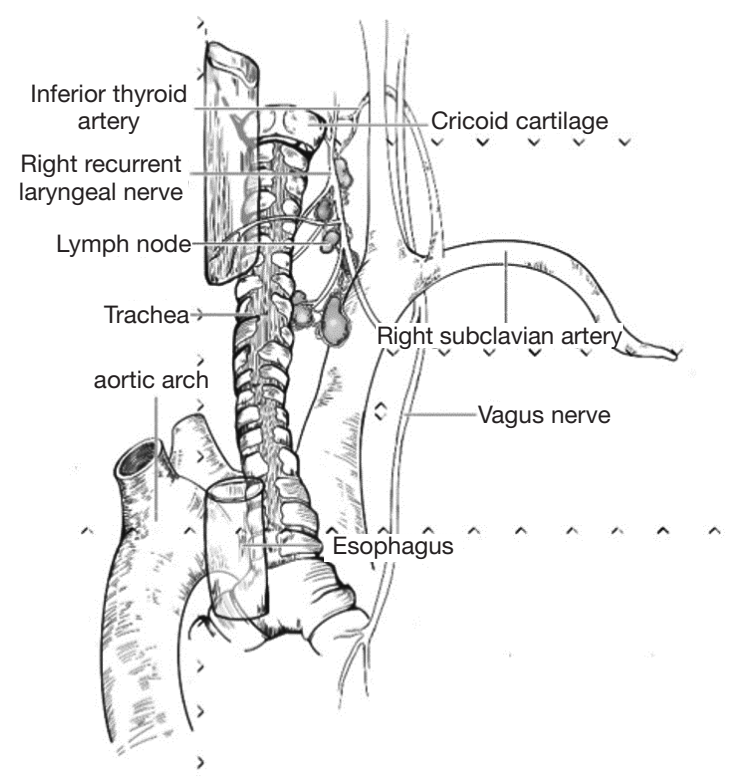

Figure 1 Anatomical sketch of right recurrent laryngeal nerve.

\section{Statistical analysis}

The data in this study were processed using SPSS Statistics Version 22.0. Categorical variables were expressed as the number of cases and percentages, and continuous variables as the median with range. Univariate analysis was tested with the $\chi^{2}$ and Mann-Whitney $U$ tests. Variables with $\mathrm{P}<0.10$, based on the univariate analysis, were included in the multivariate analysis to identify potential predictors of lymph node metastasis along the RRLN. Odds ratios (OR) and 95\% confidence intervals (CI) were expressed. Predictive values were determined by the receiver operating characteristic (ROC) curve and the cut-off value of the ROC curve was calculated as the maximum value of "sensitivity + specificity 1 ". P value $<0.05$ was considered statistically significant.

\section{Results}

\section{Patient characteristics}

A total of 307 patients, including 263 males and 44 females, were enrolled in this study. The median age was 66 (interquartile range, 61-70) years. Of these patients, $95.8 \%$ underwent minimally invasive esophagectomy, and $4.2 \%$ were treated with an open approach. The data for patient demographics, tumor location, tumor length, clinical stage, and pathologic grade are shown in Table 1.

\section{Postoperative complications}

Postoperative complications are displayed in Table 2. The overall postoperative complication rate was $22.8 \%$ (70/307). The mortality rate after 90 days was $0.3 \%$ (1/307). Vocal cord palsy (VCP) affected 14.3\% (44/307) of all patients; the incidences of right, left, and bilateral vocal cord palsy were $0.7 \%$ (2/307), $12.4 \%$ (38/307), and $1.3 \%(4 / 307)$, respectively.
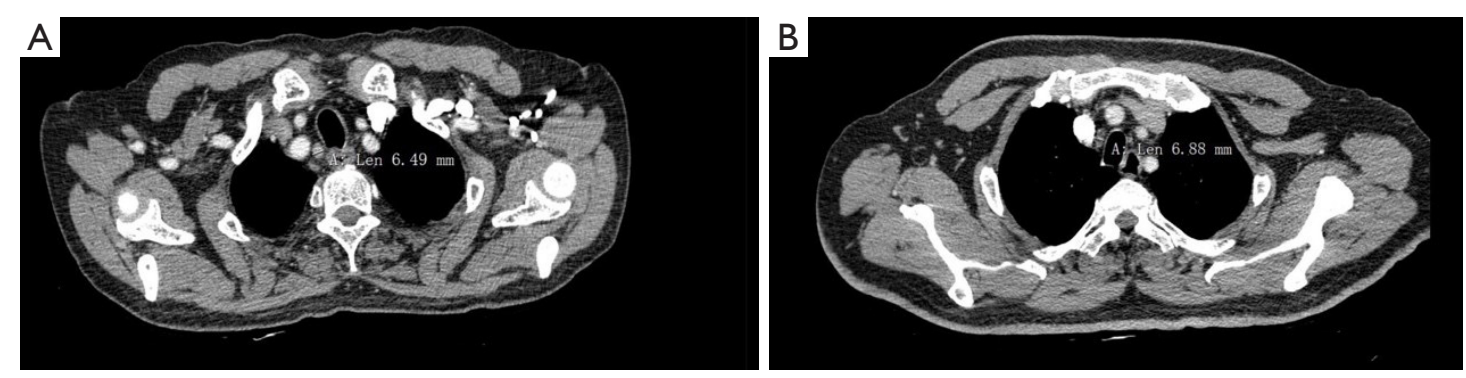

Figure 2 The short diameter of metastasis RRLN lymph nodes measured by contrast-enhanced CT scan. (A) The short diameter of metastasis lymph node was $6.49 \mathrm{~cm}$; (B) the short diameter of metastasis lymph node was $6.88 \mathrm{~cm}$. 
Table 1 Clinicopathological characteristics of patients

\begin{tabular}{|c|c|c|}
\hline Variables & Case $(n=307)$ & Rate (\%) \\
\hline Median age [year] & \multicolumn{2}{|c|}{$66[61-70]$} \\
\hline \multicolumn{3}{|l|}{ Gender } \\
\hline Male & 263 & 85.70 \\
\hline Female & 44 & 14.30 \\
\hline \multicolumn{3}{|l|}{ Tumor location } \\
\hline Upper & 39 & 12.70 \\
\hline Middle & 209 & 68.10 \\
\hline Lower & 59 & 19.20 \\
\hline \multicolumn{3}{|l|}{ Tumor length (cm) } \\
\hline$<4$ & 128 & 41.60 \\
\hline$\geq 4$ & 180 & 58.40 \\
\hline \multicolumn{3}{|l|}{ cT stage } \\
\hline $\mathrm{T} 1$ & 31 & 10.10 \\
\hline $\mathrm{T} 2$ & 158 & 51.50 \\
\hline T3 & 90 & 29.30 \\
\hline $\mathrm{T} 4$ & 28 & 9.10 \\
\hline \multicolumn{3}{|l|}{ pT stage } \\
\hline $\mathrm{T} 1$ & 66 & 21.50 \\
\hline $\mathrm{T} 2$ & 71 & 23.10 \\
\hline T3 & 159 & 51.80 \\
\hline T4 & 11 & 3.60 \\
\hline \multicolumn{3}{|l|}{ Tumor grade } \\
\hline Well & 57 & 18.60 \\
\hline Moderately & 153 & 29.80 \\
\hline Poorly & 97 & 31.60 \\
\hline \multicolumn{3}{|c|}{ Short diameter of LN [mm] } \\
\hline Negative & \multicolumn{2}{|c|}{3 [2-5] } \\
\hline Positive & \multicolumn{2}{|c|}{$4[2-6]$} \\
\hline
\end{tabular}

Continuous data are shown as median [interquartile range]. LN, lymph node.

\section{Lymph nodes dissection}

Lymph nodes were found along the RRLN in 270 patients $(87.6 \%, 270 / 307)$ by pathological study, which was much higher than was indicated by preoperative CT-scan evaluation $(57.1 \%, 176 / 307)$. In these patients, the CT detection rate of lymph nodes in patients with metastasis
Table 2 Short-term postoperative outcomes

\begin{tabular}{lcc}
\hline Characteristics & Case $(\mathrm{n}=307)$ & Rate $(\%)$ \\
\hline Complications & 70 & 22.8 \\
Respiratory system & 14 & 4.6 \\
VCP & 44 & 14.3 \\
Right VCP & 2 & 0.7 \\
Left VCP & 38 & 12.4 \\
Bilateral VCP & 4 & 1.3 \\
Leakage & 23 & 7.5 \\
90-day mortality & 1 & 0.3 \\
\hline VCP, vocal cord palsy. & &
\end{tabular}

Table 3 Comparison of CT detection rate of lymph node along RRLN

\begin{tabular}{lccc}
\hline Group & $y p N-R R L N$ & $y p N+R R L N$ & P value \\
\hline CT detection (yes) & 82 & 12 & \\
CT detection (no) & 128 & 48 & \\
Total & 210 & 60 & 0.006 \\
\hline ypN - RRLN, metastasis-free lymph node along the RRLN; ypN \\
+ RRLN, metastatic lymph node along the RRLN. CT, computed \\
tomography; RRLN, right recurrent laryngeal nerve.
\end{tabular}

was significantly higher than that in patients without metastasis (48/60 vs. 128/210, $\mathrm{P}=0.006$ ) (Table 3). Lymph node metastasis along the RRLN occurred in 60 patients (19.5\%). Of all the lymph nodes resected along the RRLN, $14.5 \%(121 / 832)$ were positive. The median short diameter of lymph nodes along the RRLN by pathological evaluation was 3 (interquartile range, 2-5) $\mathrm{mm}$ for the negative ones and 4 (interquartile range, 2-6) $\mathrm{mm}$ for the positive ones, respectively.

\section{ROC curve analysis for RRLN lymph node metastasis}

Univariate analysis revealed tumor differentiation and LNSD to be associated with lymph node metastasis along the RRLN (Table 4). In the multivariate analysis, LNSD (OR, 1.236; 95\% CI, 1.127-1.356; $\mathrm{P}<0.001$ ) was identified as the independent risk factor for RRLN lymph node metastasis.

By establishing different CT imaging cutoffs for RRLN lymph node metastasis and an LNSD ROC curve, the area under the ROC curve was found to be $0.70(\mathrm{P}<0.001)$, 
Table 4 Univariable and multivariable analysis of risk factors for lymph node metastasis of RRLN

\begin{tabular}{|c|c|c|c|c|c|c|c|}
\hline Variables & No. metastasis/total cases (\%) & \multicolumn{3}{|c|}{ Univariate analysis } & \multicolumn{3}{|c|}{ Multivariate analysis } \\
\hline Gender & & & & 0.806 & & & \\
\hline Male & $52 / 263(19.8)$ & & & & & & \\
\hline Female & 8/44 (18.2) & & & & & & \\
\hline Tumor site & & & & 0.330 & & & \\
\hline Upper & 11/39 (28.2) & & & & & & \\
\hline Middle & $39 / 209(18.7)$ & & & & & & \\
\hline Lower & $10 / 59(16.9)$ & & & & & & \\
\hline$\geq 4$ & $35 / 180(19.4)$ & & & & & & \\
\hline cT stage & & & & 0.227 & & & \\
\hline T1 & $4 / 31(12.9)$ & & & & & & \\
\hline $\mathrm{T} 2$ & 26/158 (16.5) & & & & & & \\
\hline T3 & $23 / 90(25.6)$ & & & & & & \\
\hline T4 & $7 / 28(25.0)$ & & & & & & \\
\hline Tumor grade & & & & 0.007 & & & \\
\hline Well & 6/57 (10.5) & 1.000 & & & & & \\
\hline
\end{tabular}

OR, odds ratio; $\mathrm{Cl}$, confidence interval; RRLN, right recurrent laryngeal nerve; LNSD, lymph node short diameter.

indicating that LNSD could be utilized as a diagnostic indicator of RRLN lymph node metastasis (Figure 3). An RRLN lymph node with a short diameter of $6.5 \mathrm{~mm}$ by enhanced CT imaging distinguished the presence of metastasis at this site (sensitivity $=50 \%$, specificity $=83.4 \%$ ).

\section{Discussion}

This study revised the imaging evaluation criteria for lymph node metastasis in esophageal cancer and identified that RRLNs with a short diameter of less than $10 \mathrm{~mm}$ still entail a high risk of metastasis. Through ROC analysis, an RRLN size of $6.5 \mathrm{~mm}$ was discovered to potentially be a better imaging clinical prediction standard for having metastasis at this site. The metastasis rate of lymph nodes along the
RRLN was approximate $20 \%$ and dissection of these lymph nodes may provide a survival benefit (14). This criterion could be extended to other high-risk areas of lymph node metastasis in esophageal cancer, such as the left recurrent laryngeal nerve, the paracardiac area, and the left gastric artery. It may reduce the incidence of clinical N-stage false negatives and alert clinicians to the need to address these nodes on a more routine basis.

It is clear that the current utilization of a short diameter greater than $10 \mathrm{~mm}$ is not supported as the clinical standard for CT presence of lymph node metastasis. At the same time, endoscopic resection has been increasingly used to treat superficial esophageal cancer, but with submucosal esophageal cancer often comes a high risk of lymph node metastasis $(15,16)$. With $\operatorname{sm} 2$ or $\operatorname{sm} 3$ lesions, 


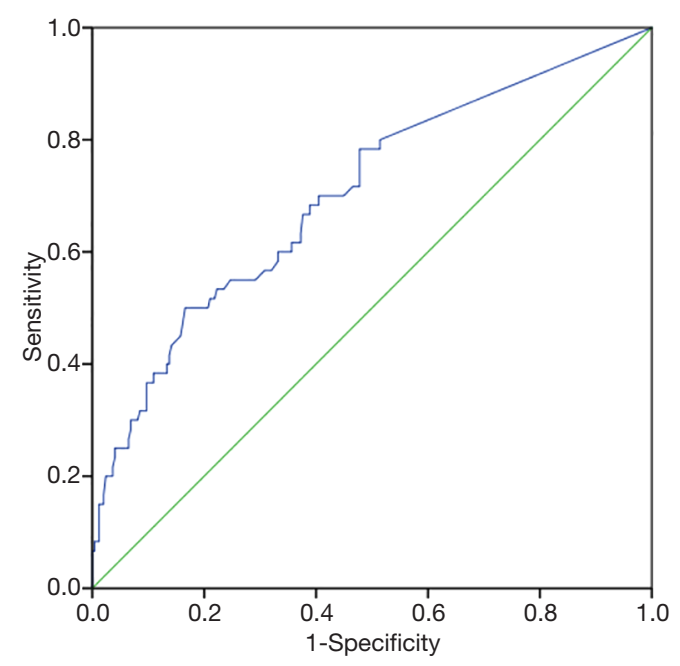

Figure 3 ROC curve of RRLN lymph node metastasis and LNSD measured by CT. ROC, receiver operating characteristic; RRLN, right recurrent laryngeal nerve; LNSD, lymph node short diameter; CT, computed tomography.

if lymphovascular invasion and large lesions (>25 $\mathrm{mm}$ ) are present, even if the lymph node does not exceed $1 \mathrm{~cm}$ in size, lymph node metastasis should still be highly suspected (17). In our study, we attempted to obtain a more reasonable evaluation system that could indicate lymph node metastasis.

In esophageal cancer, many modalities, including PET/ CT scan, EUS/FNA, and CT scan, have been used to assess lymph node metastasis. PET/CT performs excellently in detecting distant metastatic disease, although its ability to detect loco-regional disease is limited $(18,19)$. EUS/ FNA is more sensitive for lymph node examination but is technically challenging to apply in the RRLN area and its long learning curve makes it unsuitable for routine utilization (20). Therefore, we still regard imaging assessment with a high-resolution CT scan as the mainstay of lymph node evaluation, with its high availability for almost all patients.

Our study clearly demonstrated that the current detection rate of CT for lymph nodes is significantly lower than that of true pathological detection (57.14\% vs. $87.6 \%$, respectively); therefore, increasing CT sensitivity to lymph node detection is important. To achieve this, CT scanning with $1-\mathrm{mm}$ slice thickness has been suggested (21). According to the JCOG9907 trial, the CT detection rate was $48.7 \%$ in negative nodes, compared to $72.7 \%$ in positive nodes (22). In our study, CT scanning found 61\% (128/210) of negative nodes, as well as $80 \%$ (48/60) of positive. Therefore, the CT scanning still has a good detection value for metastatic lymph nodes. However, a better criteria is required.

In our study, tumor differentiation and LNSD were found to be associated with lymph node metastasis in the RRLN region, which provides guidance for patients who require routine dissection of RRLN lymph nodes. For patients with superficial lesions, CT findings at the RRLN site may alter plans for endoscopic submucosal dissection (ESD). If surgery is not tolerated, biopsy of nodes in the RRLN location should be considered, especially nodes $>6.5 \mathrm{~mm}$ in size. Although lymph node biopsy is difficult to conduct in the thoracic outlet area, it can still be achieved under general anesthesia. In our study, a short diameter of $6.5 \mathrm{~mm}$ in the RRLN lymph node on CT images was considered to be the cutoff value for predicting metastasis. Ideally, CT lymph node size should be correlated with other pathologic features of the tumor to predict metastasis and help guide proper management of superficial ESCC.

Despite our findings, this study had some limitations. Firstly, the preoperative CT detection rate of lymph nodes at the RRLN site was not high enough, and we had no way to study all of the lymph nodes. Secondly, some patients had multiple RRLN lymph nodes, and we could only take the largest one into account to calculate LNSD, although the proportion of these was small. Finally, the preoperative CT diagnosis of lymph nodes may not correspond to the proper site of surgical removal and postoperative pathological diagnosis of lymph nodes.

In summary, patients with thoracic ESCC have a high rate of lymph node metastasis along the RRLN. This study found that LNSD $\geq 6.5 \mathrm{~mm}$ on CT imaging should serve as a clinical predictor of lymph node metastasis at the RRLN site. Furthermore, the larger the short diameter is, the higher the risk of metastasis. The discoveries made in this study consistute important progress, although more improvements need to be made in the pretherapeutic prediction of ESCC.

\section{Acknowledgments}

Funding: This study was funded by grants from Three years of clinical innovation action plan (16CR1035B) and Shanghai Municipal Education Commission-Gaofeng Clinical Medicine Grant Support.

\section{Footnote}

Reporting Checklist: The authors have completed the STARD 
reporting checklist. Available at http://dx.doi.org/10.21037/ atm-20-4991

Data Sharing Statement: Available at http://dx.doi. org/10.21037/atm-20-4991

Conflicts of Interest: All authors have completed the ICMJE uniform disclosure form (available at http://dx.doi. org/10.21037/atm-20-4991). The authors have no conflicts of interest to declare.

Ethical Statement: The authors are accountable for all aspects of the work in ensuring that questions related to the accuracy or integrity of any part of the work are appropriately investigated and resolved. This study was approved by the Ethics Committee of Shanghai Chest Hospital (No. KS1734). The study was conducted in accordance with the Declaration of Helsinki (as revised in 2013). Because of the retrospective nature of the research, the requirement for informed consent was waived.

Open Access Statement: This is an Open Access article distributed in accordance with the Creative Commons Attribution-NonCommercial-NoDerivs 4.0 International License (CC BY-NC-ND 4.0), which permits the noncommercial replication and distribution of the article with the strict proviso that no changes or edits are made and the original work is properly cited (including links to both the formal publication through the relevant DOI and the license). See: https://creativecommons.org/licenses/by-nc-nd/4.0/.

\section{References}

1. Siegel RL, Miller KD, Jemal A. Cancer statistics, 2020. CA Cancer J Clin 2020;70:7-30.

2. Chen $W$, Zheng R, Baade PD, et al. Cancer statistics in China, 2015. CA Cancer J Clin 2016;66:115-32.

3. Tachimori Y, Ozawa S, Numasaki H, et al. Efficacy of lymph node dissection by node zones according to tumor location for esophageal squamous cell carcinoma. Esophagus 2016;13:1-7.

4. Ganesamoni S, Krishnamurthy A. Three-field transthoracic versus transhiatal esophagectomy in the management of carcinoma esophagus-a single--center experience with a review of literature. J Gastrointest Cancer 2014;45:66-73.

5. van der Horst S, de Maat MFG, van der Sluis PC, et al. Extended thoracic lymph node dissection in robotic- assisted minimal invasive esophagectomy (RAMIE) for patients with superior mediastinal lymph node metastasis. Ann Cardiothorac Surg 2019;8:218-25.

6. Ye K, Xu JH, Sun YF, et al. Characteristics and clinical significance of lymph node metastases near the recurrent laryngeal nerve from thoracic esophageal carcinoma. Genet Mol Res 2014;13:6411-9.

7. Kato H, Tachimori Y, Mizobuchi S, et al. Cervical, mediastinal, and abdominal lymph node dissection (threefield dissection) for superficial carcinoma of the thoracic esophagus. Cancer 1993;72:2879-82.

8. Noshiro H, Iwasaki H, Kobayashi K, et al. Lymphadenectomy along the left recurrent laryngeal nerve by a minimally invasive esophagectomy in the prone position for thoracic esophageal cancer. Surg Endosc 2010;24:2965-73.

9. Hulscher JB, van Sandick JW, de Boer AG, et al. Extended transthoracic resection compared with limited transhiatal resection for adenocarcinoma of the esophagus. $\mathrm{N} \mathrm{Engl} \mathrm{J}$ Med 2002;347:1662-9.

10. Min YW, Lee H, Song BG, et al. Comparison of endoscopic submucosal dissection and surgery for superficial esophageal squamous cell carcinoma: a propensity score-matched analysis. Gastrointest Endosc 2018;88:624-33.

11. Mannath J, Ragunath K. Role of endoscopy in early oesophageal cancer. Nat Rev Gastroenterol Hepatol 2016;13:720-30.

12. van Vliet EP, Heijenbrok-Kal MH, Hunink MG, et al. Staging investigations for oesophageal cancer: a metaanalysis. Br J Cancer 2008;98:547-57.

13. Low DE, Alderson D, Cecconello I, et al. International Consensus on Standardization of Data Collection for Complications Associated With Esophagectomy: Esophagectomy Complications Consensus Group (ECCG). Ann Surg 2015;262:286-94.

14. Tachimori Y, Ozawa S, Numasaki H, et al. Efficacy of lymph node dissection by node zones according to tumor location for esophageal squamous cell carcinoma. Esophagus 2016;13:1-7.

15. Gockel I, Sgourakis G, Lyros O, et al. Risk of lymph node metastasis in submucosal esophageal cancer: a review of surgically resected patients. Expert Rev Gastroenterol Hepatol 2011;5:371-84.

16. Tachibana M, Kinugasa S, Shibakita M, et al. Surgical treatment of superficial esophageal cancer. Langenbecks Arch Surg 2006;391:304-21.

17. Weksler B, Kennedy KF, Sullivan JL. Using the National 
Cancer Database to create a scoring system that identifies patients with early-stage esophageal cancer at risk for nodal metastases. J Thorac Cardiovasc Surg 2017;154:1787-93.

18. Chang KY, Chang JY, Chao J, et al. Modern staging and utility of PET imaging in esophageal cancer management. J Natl Compr Canc Netw 2008;6:862-9.

19. Li B, Li N, Liu S, et al. Does [18F] fluorodeoxyglucosepositron emission tomography/computed tomography have a role in cervical nodal staging for esophageal squamous cell carcinoma? [published online ahead of print, 2019 Dec 11. J Thorac Cardiovasc Surg

Cite this article as: Li B, Li B, Jiang H, Yang Y, Zhang X, Su Y, Hua R, Gu H, Guo X, Ye B, Yang Y, He Y, Sun Y, Piessen G, Hochwald SN, Cuesta MA, Birdas TJ, Li Z; written on behalf of the AME Thoracic Surgery Collaborative Group. The value of enhanced CT scanning for predicting lymph node metastasis along the right recurrent laryngeal nerve in esophageal squamous cell carcinoma. Ann Transl Med 2020;8(24):1632. doi: 10.21037/atm-20-4991
2019:S0022-5223(19)33960-1.

20. Vazquez-Sequeiros E, Norton ID, Clain JE, et al. Impact of EUS-guided fine-needle aspiration on lymph node staging in patients with esophageal carcinoma. Gastrointest Endosc 2001;53:751-7.

21. Rice TW. Clinical staging of esophageal carcinoma. CT, EUS, and PET. Chest Surg Clin N Am 2000;10:471-85.

22. Yokota T, Igaki H, Kato K, et al. Accuracy of preoperative diagnosis of lymph node metastasis for thoracic esophageal cancer patients from JCOG9907 trial. Int J Clin Oncol 2016;21:283-8. 